\title{
Decreased capacity for mental effort after single supratentorial lacunar infarct may affect performance in everyday life
}

\author{
M J E Van Zandvoort, L J Kappelle, A Algra, E H F De Haan
}

\begin{abstract}
Objectives-The long term outcome after a single symptomatic lacunar infarct may be less favourable than is generally assumed. Patients often present with complaints such as fatigue or "being different from before the stroke", for which there are no obvious physical explanations. Although cognitive functioning is considered normal in most patients with lacunar infarction in the internal capsule or corona radiata, a study was carried out to determine if subclinical changes in mental or emotional function can explain these vague complaints characteristic for their disablement.
\end{abstract}

Methods-Sixteen patients, each with a single symptomatic supratentorial lacunar infarct, and 16 matched healthy controls were examined with an extensive neuropsychological screening battery and a standardised questionnaire aimed at emotional problems. The mean number of correct responses was calculated for each subject and averaged within each group.

Results-Although, on the whole, there were no differences in performance, patients' results on the following tasks in different modalities showed evidence for decreased performance under relatively more demanding conditions: line orientation task (mean difference (MD) $261 \mathrm{~ms}$; $95 \%$ confidence interval (95\%CI) 94 to 428$)$, Rey-Osterrieth delayed recall (MD-3.8, $95 \%$ CI -7.5 to 0.0 ), visual elevator subtest of the everyday attention task (EAT) (MD $-0.7,95 \% \mathrm{CI}-1.5$ to 0.1 ), lottery subtest of the EAT (MD $-0.6,95 \% \mathrm{CI}-1.3$ to 0.1 ) and WAIS similarities (MD $-3.295 \% \mathrm{CI}-6.3$ to 0.1). Patients also more often had emotional disturbances than controls.

Conclusion-Both subtle cognitive impairments and emotional disturbances may play a part in the decreased compe- tence in everyday life of patients with a supratentorial lacunar infarct.

(F Neurol Neurosurg Psychiatry 1998;65:697-702)

Keywords: lacunar infarction; neuropsychology; subcortical infarction

Sixty per cent of patients with stroke have an impaired quality of life (QOL) after six months. ${ }^{1}$ Both physical and cognitive impairments may have detrimental effects on daily living. In the past decade, the association of cognitive impairments and QOL after stroke has received growing interest. ${ }^{2}$

Some $25 \%$ of strokes consist of lacunar infarcts in the deep regions of the brain. Over 20 different kinds of lacunar syndromes have been reported, all of which emphasised sparing of cognitive functioning. ${ }^{3}$ This is not surprising, as the affected brain areas are not normally associated with higher mental function, as long as the thalamus and the caudate nucleus are not involved. ${ }^{4}$ The prognosis of a lacunar infarct seems favourable for physical and cognitive functioning in comparison with other types of stroke, ${ }^{6}$ but reduction of QOL has been seen also after lacunar infarction. ${ }^{1} 78$

Although cognitive impairment not considered a characteristic of lacunar syndrome, some reports describe individual cases with remarkable focal neuropsychological impairments (table 1). Suboptimal functioning after a lacunar infarct without an explanation has also been reported. ${ }^{2}$ In clinical practice patients with single lacunar infarcts often report non-specific symptoms on long term follow up. Although recovered physically quite well, they may continue to report certain discomfort described as fatigue, "being different from before the stroke", "things not going as they used to", etc. These complaints are in many cases confirmed by relatives. We posited that mild cognitive dysfunctioning after a single

Table 1 Neuropsychological impairments after a single lacunar infarct: case studies in the literature

Utrecht University,

The Netherlands

A Algra

Correspondence to:

Dr MJE van Zandvoort,

Psychological Laboratory,

University of Utrecht,

Heidelberglaan 2, 3584 CS

Utrecht, The Netherlands.

Telephone 0031302534281 ;

fax 003130 2534511; email

m.vanzandvoort@FSS.uu.NI

Received 2 December 1997

and in final form

29 April 1998

Accepted 13 May 1998 
Table 2 Semistructured interview on the status after the lacunar infarct

1 Do you suffer from sleeping disturbances after the occurrence of your lacunar infarct? 2 Are you more rapidly fatigued after the occurrence of your lacunar infarct?

3 Are you more rapidly fatigued after the occurrence of your lacunar infarct?

4 Do you feel you are more easily emotional after the occurrence of your lacunar infarct? 5 Do you feel more rapidly irritated after the occurrence of your lacunar infarct?

lacunar infarct might be the cause of these symptoms and might, in turn, negatively influence the QOL. In addition we looked at the presence of emotional disturbances as an alternative or additive explanation.

\section{Patients and methods}

Patients were recruited from a group of 21 who had been treated for a lacunar syndrome due to a single, first ever lacunar infarct, at the University Hospital of Utrecht. The interval between the occurrence of the lacunar infarct and the examination ranged between 6 and 45 months, with a mean of 14 (SD 11) months. In all patients the infarct had been visible on CT or MRI, in the centrum semiovale, internal capsule, or corona radiata. Patients with involvement of the thalamus or the caudate nucleus were excluded, as were patients with abnormalities on CT or MRI other than the single lacunar infarct. Moreover, patients needed to be able to communicate in Dutch. They were also younger than 75 years of age and had no history of any other form of cardiovascular disease, brain damage, or any other background condition that could influence cognitive function, such as a psychiatric disorder or alcoholism. For each patient, a healthy control subject of similar age, sex, and education was sought. As a result, the two groups were highly comparable for demographic variables. The patients were reimbursed for travel expenses and the control subjects were paid a fixed sum for their participation. The study protocol was approved by the ethics committee of the hospital (WHO standard) and all patients filled out an informed consent form.

The testing procedure was identical for patients and controls, and took about 3 hours. After a short introduction the Barthel index was completed and the patients were asked to answer "yes" or "no" to questions from a semistructured interview about emotional functioning, irritability, sleeping disturbances, fatigue, and concentration disturbances (table 2 ). ${ }^{9}$ The

Table 3 The 17 neuropsychological tests in the order in which they were administered to both the controls and the patients

\begin{tabular}{ll}
\hline Neuropsyhological tests & Cognitive domain \\
\hline Everyday attention task & Attention \\
Boston naming task & Language \\
Token test & Language \\
Digit span forward and backward & Working memory and attention (verbal) \\
Corsi blocks test & Working memory and attention (non-verbal) \\
WAIS similarities & Executive functioning \\
Verbal fluency (UNCA) & Language and executive functioning \\
Category fluency & Language and executive functioning \\
& Language \\
Chapman reading task & Verbal memory (learning and short term memory) \\
Rey auditory verbal learning task (RAVLT) & \\
Rey-Osterieth complex figure & Perception and construction \\
(copy and direct recall) & Perception and construction \\
Line orientation (computerised) & Motor function \\
Motor reaction task (simple and complex) & Executive functioning \\
Trailmaking A and B & Verbal memory (long term memory) \\
RAVLT delayed recall and recognition & Non-verbal memory (long term memory) \\
Rey-o delayed recall & Perception and construction \\
WAIS block design &
\end{tabular}

controls were asked the same questions but they were also asked to compare their present state with that 1 year previously. Subsequently, a broad screening of the main areas of cognitive functioning was performed. The order of the tests was identical for all subjects; all subjects had a break at the same point in the procedure. A total of 17 tests was used to assess perception and construction, memory, language, attention and executive function, and motor behaviour (table 3). The different cognitive domains were studied as follows:

\section{PERCEPTION AND CONSTRUCTION}

The computerised line orientation is based on the line orientation task of Benton. ${ }^{10}$ Two lines are presented below each other on the screen and the subject is asked to judge as fast as possible whether the lines are parallel or not. During this task the reaction time is recorded. The ReyOsterrieth complex figure task requires subjects to copy a complex abstract line figure..$^{10}$ In the WAIS block design, the subject is asked to reproduce a line drawing under time pressure by means of a number of identical blocks. ${ }^{10}$

\section{MEMORY}

The digit span was used to assess verbal working memory. In this test increasing sequences of numbers have to be repeated in the same order as they were presented orally. ${ }^{10}$ Nonverbal memory was tested in the same way with the Corsi blocks test, ${ }^{10}$ in which an increasing sequence of positions has to be reproduced in the correct order. Verbal memory was assessed by means of the Rey auditory verbal memory task, in which the subject is asked to learn 15 non-related words over five auditory presented trials. The long term memory was tested in the delayed recall and recognition after $30 \mathrm{~min}-$ utes. The Rey-Osterrieth complex figure, direct and delayed, recall measures the incidental short and long term non-verbal memory. ${ }^{10}$ The abstract line drawings from the Rey-Osterrieth copy have to be recalled after 1 and 30 minutes.

\section{LANGUAGE}

Sixty line drawings of growing complexity of the Boston naming task was presented for a naming response. ${ }^{10}$ The token test was used to evaluate the understanding of spoken language. ${ }^{10}$ The Chapman reading task assessed reading comprehension under time constraints. In 21 sentences subjects have to detect one word that is semantically inappropriate. ${ }^{11}$ On the letter fluency task subjects are asked to name as many words as they can think of starting with a $\mathrm{U}, \mathrm{N}$, $\mathrm{C}$, or A. ${ }^{10}$ Category fluency followed the same procedure but animals and professions had to be named. ${ }^{10}$

\section{ATTENTION AND EXECUTIVE FUNCTION}

The task of everyday attention (TEA) consists of six subtests assessing various aspects of attention representing mental flexibilities, such as sustained attention, distractibility, directed attention, and attentional shifting. ${ }^{12}$ The test is based on attentional demands in the daily living. In the trailmaking A numbers from 1-25 are distributed on a paper and must be connected in the right order with a pencil, in 
Table 4 Summaries of the biographical and clinical characteristics of patients

\begin{tabular}{|c|c|c|c|c|c|c|}
\hline & Sex & Age & $\begin{array}{l}\text { Education } \\
\text { level }\end{array}$ & Lesion & Side & $\begin{array}{l}\text { Interval } \\
I-E\end{array}$ \\
\hline 1 & $\mathrm{~F}$ & 67 & 6 & cor rad & $\mathrm{L}$ & 20 \\
\hline 2 & $\mathrm{~F}$ & 29 & 5 & c semi ov & $\mathrm{L}$ & 6 \\
\hline 3 & $M$ & 40 & 6 & cor rad & $\mathrm{L}$ & 10 \\
\hline 4 & $M$ & 53 & 4 & post int caps & $\mathrm{L}$ & 17 \\
\hline 5 & $\mathrm{M}$ & 57 & 4 & post int caps & $\mathrm{R}$ & 45 \\
\hline 6 & $M$ & 45 & 7 & post int caps & $\mathrm{L}$ & 8 \\
\hline 7 & M & 62 & 6 & cor rad & $\mathrm{L}$ & 8 \\
\hline 8 & $\mathrm{~F}$ & 61 & 6 & ant int caps & $\mathrm{R}$ & 27 \\
\hline 9 & M & 49 & 4 & cor rad & $\mathrm{L}$ & 6 \\
\hline 10 & $\mathrm{M}$ & 76 & 4 & ant int caps & $\mathrm{L}$ & 11 \\
\hline 11 & $\mathrm{~F}$ & 55 & 3 & cor rad & $\mathrm{R}$ & 6 \\
\hline 12 & $\mathrm{~F}$ & 60 & 5 & post int caps & $\mathrm{R}$ & 8 \\
\hline 13 & $M$ & 63 & 2 & cor rad & $\mathrm{L}$ & 6 \\
\hline 14 & $\mathrm{~F}$ & 72 & 7 & post int caps & $\mathrm{L}$ & 20 \\
\hline 15 & $M$ & 50 & 6 & cor rad & $\mathrm{R}$ & 25 \\
\hline 16 & $\mathrm{~F}$ & 70 & 7 & subins & $\mathrm{L}$ & 7 \\
\hline
\end{tabular}

Level of education as described by Verhage and Van der Werff. ${ }^{27} \mathrm{c}$ semi ov=centrum semi ovale; cor rad=corona radiata; post int caps=posterior limb of the internal capsule; ant int caps=anterior limb of the internal capsule; int caps=internal capsule; side=hemisphere L (left) R (right); interval I-E=interval between the infarct and the examination presented in months.

part $\mathrm{B}$ letters have to be connected in alphabetic order. ${ }^{10}$ In $\mathrm{C}$ a combination is made of $\mathrm{A}$ and $\mathrm{B}$, in that the letters and numbers have to be alternately connected, both in the right order. Concept formation and abstract reasoning was assessed by means of the WAIS similarities verbal test.

MOTOR FUNCTION

The computerised motor reaction task assesses the reaction time of the correct responses and the movement rate in a simple and complex form. This task is integrated into the research

Table 5 Means (SD) and mean differences (95\% CI) for the neuropsychological tests for both groups

\begin{tabular}{|c|c|c|c|c|}
\hline \multirow[b]{2}{*}{ Neuropsychological test } & \multicolumn{2}{|l|}{ Group mean } & \multirow{2}{*}{$\begin{array}{l}\text { Mean } \\
\text { difference }\end{array}$} & \multirow[b]{2}{*}{$95 \% C I$} \\
\hline & Patients (SD) & Controls (SD) & & \\
\hline \multicolumn{5}{|l|}{ Perception and construction: } \\
\hline Line orientation $(\mathrm{ms})$ & $813(212)$ & $552(251)$ & 261 & 94 to 428 \\
\hline Rey-Osterrieth copy (rs) & $35.3(1.3)$ & $35.5(0.7)$ & -0.25 & -1.0 to 0.5 \\
\hline WAIS block design (rs) & $14.6(6.0)$ & $17.3(5.6)$ & -2.6 & -6.9 to 1.6 \\
\hline \multicolumn{5}{|l|}{ Memory: } \\
\hline Digit span forward (nc) & $5.8(1.2)$ & $5.7(1.2)$ & 0.0 & -0.9 to 0.9 \\
\hline Digit span backward (nc) & $4.5(1.0)$ & $4.5(1.0)$ & 0.1 & -0.7 to 0.8 \\
\hline Corsi blocks test (nc) & $5.5(1.0)$ & $4.9(0.8)$ & 0.6 & 0.1 to 1.2 \\
\hline \multicolumn{5}{|l|}{ RAVLT: } \\
\hline Total trials (nc) & $42.7(9.5)$ & $42.5(9.9)$ & 3.5 & -6.9 to 7.4 \\
\hline Delayed recall (nc) & $9.1(2.7)$ & $8.8(3.3)$ & 0.3 & -2.0 to 2.5 \\
\hline Recognition (nc) & $28.8(1.8)$ & $29.5(0.6)$ & -0.8 & -1.8 to 0.2 \\
\hline \multicolumn{5}{|l|}{ Rey-Osterrieth: } \\
\hline Direct recall (rs) & $22.6(5.4)$ & $25.7(5.5)$ & -3.0 & -7.1 to 0.9 \\
\hline Delayed recall (rs) & $21.7(5.2)$ & $25.5(4.9)$ & -3.8 & -7.5 to 0.0 \\
\hline \multicolumn{5}{|l|}{ Language: } \\
\hline Boston naming task (nc) & $56(2.9)$ & $59(1.6)$ & -2.6 & -4.4 to -0.9 \\
\hline Tokentest (nc) & $19(1.7)$ & $20(1.7)$ & -1.0 & -2.3 to 0.3 \\
\hline Chapman reading task (nc) & $16.8(3.6)$ & $17.9(4.6)$ & -1.1 & -4.2 to 2.0 \\
\hline Letter fluency (nc) & $40.3(14)$ & $41.7(14.8)$ & -1.4 & -12 to 9.2 \\
\hline Category fluency (nc) & $41.3(11.5)$ & $44(10.9)$ & -2.7 & -10.9 to 5.5 \\
\hline \multicolumn{5}{|l|}{ Attention and executive function: } \\
\hline \multicolumn{5}{|l|}{ EAT: } \\
\hline Mapsearch (nc) & $62.5(11)$ & $65(14)$ & -2.5 & -12.5 to 7.4 \\
\hline Telesearch (s) & $3.7(1.0)$ & $3.3(0.9)$ & 0.4 & -0.3 to 1.1 \\
\hline Visual elevator (nc) & $8.9(1.4)$ & $9.6(0.6)$ & -0.7 & -1.5 to 0.1 \\
\hline Elevator counting (nc) & $6.6(1.4)$ & $6.8(0.6)$ & 0.4 & -1.0 to 0.6 \\
\hline Elevator reversal (s) & $4.4(2.9)$ & $6.1(3.3)$ & -1.7 & -4.1 to 0.7 \\
\hline Lottery (nc) & $9(1.1)$ & $9.6(0.7)$ & -0.6 & -1.3 to 0.1 \\
\hline \multicolumn{5}{|l|}{ Trailmaking: } \\
\hline Part A (s) & $37.4(9.1)$ & $32.9(13.2)$ & 4.4 & -3.8 to 12.7 \\
\hline Part B (s) & $41.9(15.4)$ & $37.5(23.6)$ & 4.4 & -10.2 to 19 \\
\hline Part C (s) & $67.1(30.6)$ & $51.5(25)$ & 15.6 & -5.2 to 36 \\
\hline WAIS similarities (rs) & $18.2(4.5)$ & $21.3(4.2)$ & -3.2 & -6.3 to -0.1 \\
\hline \multicolumn{5}{|l|}{ Motor function: } \\
\hline MPT simple (ms) & $344(60)$ & $344(54)$ & 0.1 & -42 to 42 \\
\hline MPT complex (ms) & $394(70)$ & $391(94)$ & 3.5 & -58 to 65 \\
\hline
\end{tabular}

$\mathrm{rs}=$ Raw score; $\mathrm{nc}=$ number correct. software package MINDS. ${ }^{13}$ The subject is asked to react on a flashing light on a button box consisting of one red and five white buttons. During the whole test the red button must be held down and only when one of the other lights flashed, the finger has to be switched from the red button or to be moved from the red button to press one of the other five buttons.

For most of the above tests the mean number of correct responses was calculated for the patient and control groups. The WAIS subtasks, the Rey-Osterrieth task, and the EAT map search used raw scores. The motor reaction time task, the line orientation, the trailmaking, and the EAT telesearch used reaction time ; the median reaction time was calculated for each subject and subsequently averaged within each group. These data were analysed with Student's $t$ test for independent samples and tested with a $95 \%$ confidence interval $(95 \%$ CI) by means of SPSS 7.0. In addition, three post hoc analyses were carried out; firstly, a within group analysis to compare patients with left and patients with right hemispheric lesions by means of Student's $t$ test for independent samples. A second post hoc analysis evaluated the effect of the intrahemispheric lesion localisation on the test performance by Student's $t$ test for independent samples. We distinguished between lesions in either the internal capsule or the corona radiata. Finally, we studied the influence of the elapsed time between the onset of the stroke and the moment of neuropsychological screening by relating the elapsed time with the test performances.

\section{Results}

Five of the 21 patients contacted by telephone declined to participate for personal reasons that did not influence the selection criteria. The remaining 16 patients received a written confirmation and further information by mail. Of these patients, seven had a lacune in the internal capsule, seven in the corona radiata, one in the centrum semiovale, and one in the subinsula region (table 4). Fifteen patients were right handed, one was ambidextrous; all control subjects were right handed. The biographical and clinical characteristics are shown in table 4 . The results of the patient and control groups were free of statistical outliers (all data fell within 2 SD of the group mean) suggesting that the overall results were not disproportionately influenced by the test performance of one particular patient or control.

Table 5 shows the differences for the neuropsychological tests between the patients and the controls. There were no significant differences in performance of most tasks. However, patients had more problems in judging whether two lines were oriented in the same direction or not (mean difference (MD) $261 \mathrm{~ms}, 95 \%$ confidence interval (95\% CI) 94 to 428). Both groups performed similarly on the digit span test for short term verbal memory and on the Corsi blocks test for non-verbal short term memory the patients performed slightly better that the control subjects (MD 0.6, 95\% CI 0.1 to 1.2 ). Patients and controls were equally proficient in copying the Rey-Osterrieth complex 
Table 6 Changes in emotional functioning, irritability, concentration, fatigue, and sleeping before and after the lacunar infarct (patients) and during the past year (controls)

\begin{tabular}{lllll}
\hline & $\begin{array}{l}\text { Patients } \\
(n=16)\end{array}$ & $\begin{array}{l}\text { Controls } \\
(n=16)\end{array}$ & $\begin{array}{l}\text { Prevalence } \\
\text { ratio }\end{array}$ & $95 \%$ CI \\
\hline Emotional functioning & $12(75)$ & $2(12)$ & 6 & $1.6-23$ \\
Irritability & $6(38)$ & $4(25)$ & 1.5 & $0.5-4.3$ \\
Concentration & $7(46)$ & $4(25)$ & 1.75 & $0.6-4.8$ \\
Fatigue & $6(38)$ & $2(12)$ & 3 & $0.7-12.7$ \\
Sleeping disturbances & $4(25)$ & $4(25)$ & 1 & $0.3-3.3$ \\
\hline
\end{tabular}

figure, but the patients' performance decreased on the immediate recall after about 2 minutes ( $95 \% \mathrm{CI}-7.1$ to 0.9 ), and after a 30 minute delay recall of the patient group was significantly below that of the controls $(95 \%$ CI -7.5 to -0.0). By contrast, the patients performed entirely normally on long term verbal memory task (Rey auditory verbal learning task).

There were no differences on the language tasks apart from a significant difficulty in naming objects from line drawings for the patients on the Boston naming task (MD -2.6, 95\% CI-4.4 to -0.9$)$. The subtest visual elevator of the TEA showed that the patients had problems with alternately counting back and forth, while simultaneously monitoring the score under time pressure (MD -0.7, 95\% CI -1.5 to 0.1 ). The lottery subtest of the EAT, a vigilance task, showed that the patients had more difficulty in staying alert while listening to a boring voice on a tape for 10 minutes (MD $-0.6,95 \% \mathrm{CI}-1.3$ to 0.1 ). The patients had more difficulties on the WAIS Similarities (MD $-3.2,95 \%$ CI -6.3 to -0.01 ). No significant differences were found on the simple and complex reaction time test.

The five patients with lacunes in the right hemisphere and the 11 patients with lacunes in the left hemisphere had similar age and education. The results of Student,s $t$ test for independent pairs showed no differences between the two groups for most tasks. However, on the category fluency and letter fluency the performance of the patients with left hemispheric lesions tended to be lower compared with the patients with right hemisphere lesions (MD -9.2, 95\% CI -18.7 to 0.4 and MD 14.7, $95 \% \mathrm{CI}-32.4$ to 3.0 respectively). On the line orientation task the difference was even more pronounced: the right hemispheric patients performed significantly below the left hemispheric patients (MD $-265,95 \%$ CI -524 to $-6.3)$. The analysis to evaluate the influence of the intrahemispheric lesion localisation on the test performance disclosed no significant differences between the patients with lesions in the internal capsule or in the corona radiata. Finally, there was no relation between the time elapsed between stroke onset and the moment of neuropsychological testing and the performance on the various tests.

The Barthel index disclosed a maximum score for all patients at the time of the neuropsychological screening. On average, patients reported a change of emotional functions somewhat on all the questions of the semistructured interview more often than controls. This difference was significant for emotional functioning (table 6) (prevalence ratio 6, $95 \%$ CI 1.6 to 22.6 ).

\section{Discussion}

The aim of this study was to explore the possibility of neuropsychological impairments after a single lacunar stroke in a cerebral hemisphere. As expected, we found a normal performance on most tasks, indicating that this group of patients does not have marked cognitive impairments or general cognitive deterioration. However, on six of the tests the patients' performances were significantly reduced compared with those of the control subjects.

Superficial inspection of the differences suggests a rather random set of tasks that posed problems for the patients. However, on closer examination a pattern can be discerned. It seems that all these tasks were particularly strenuous, independently of the specific mental function evaluated. Of all the memory tasks used, the Rey-Osterrieth task caused difficulties, because this is the only memory task employing an incidental learning procedure. That is, subjects are not forewarned that their memory will be tested. Our interpretation is that if the patients are not explicitly told to remember, they do not pay enough attention to store the stimuli automatically, as is the case for normal controls on this kind of task. Attentional difficulties became apparent on two separate subtests of the everyday attention task. Patients were less able to stay alert on a vigilance task and had more problems in concept. On the Benton line orientation task patients were as proficient as controls in judging correctly the orientation of both presented lines. The specific problem became apparent in the reaction time; they gave the correct answer, but it took them longer to judge than the controls. As the available time to give the correct answer is limited, some of correct answers were out of the scoring range. It seemed as if the attention capacity under time pressure was disproportionately limited for patients. A decreased performance for the patients was also found on the WAIS similarity test. This task is associated with executive functioning and abstract thinking. Both functions are not directly associated with a particular capacity but with an effective use of several capacities, as are needed in problem solving and planning.

Some patients used antihypertensive drugs. The impact of these on neuropsychological functioning has been reviewed by Dimsdale et $a l,{ }^{14}$ and they found no meaningful effect on the various cognitive domains. If there is a negative effect, this will be found on perceptual motor skills and our data do not show any difficulties in this cognitive domain. Therefore, we do not expect interference of the effect of antihypertensive drugs in our results.

The cognitive impairments found in patients with a single symptomatic lacunar infarct seem not to be a problem of competence but of performance and can be formulated in terms of mental effort as described by Kahneman. ${ }^{15}$ The attentional capacity is limited. Attentional effort reflects the allocation of cognitive and behavioural resources. The patients have to invest more effort to reach the same level of performance as controls. This is not a problem on low demanding tasks but as soon as the task 
or the context becomes more strenuous, performance decreases. The significant group difference on the Boston naming task, assessing word finding abilities, is the only task that does not fit our interpretation and may be caused by chance. However, language impairment due to lesions in the internal capsule has been reported, but these patients often have more extensive lesions involving more than one structure. ${ }^{16-19}$ Although we are aware of the fact that we must interpret our data with caution due to the relative small group sizes, we are encouraged by the consistency of the discriminating tasks leading to a pattern pointing towards problems with mental effort.

The first post hoc analysis showed a lateralisation effect. The comparison between patients with a lacunar infarct in the dominant hemisphere or in the non-dominant hemisphere, showed a lateralisation effect in line with cortical lateralisation of function. Left hemispheric lacunes seem to cause more problems on language tasks, and right hemispheric lacunes on visuospatial tasks. We thus infer that a single supratentorial lacunar infarct can give rise to problems of mental effort with subclinical neuropsychological deficits. The time elapsed between the stroke onset and the moment of neuropsychological screening had no influence on the test performance, and post hoc analysis to compare the intrahemispheric lesion localisation by means of the gross dichotomy did not approach significance either.

Neuropsychological deficits after a subcortical infarct have been described before in case reports (table 1). Impairments in the domain of language, memory, visuospatial functioning, attention, and concentration are reported after a single lacunar infarct, but there does not seem to be a consistent pattern between the involved structures and the neuropsychological impairments. In all the reported case studies involvement of adjacent nuclei, such as the thalamus or the nucleus caudatus, was excluded as an explanation for the findings. Various other underlying mechanisms, such as compression of adjacent structures or disconnection of important fibre pathways, were invoked. The uniqueness of the various reported case studies and the different research methods make a valid comparison between the studies difficult. In most studies a patient was subjected to post hoc investigation to show the noticeable impairment, and not subjected to broad neuropsychological screening. By contrast, in our study a group of patients was tested for a range of mental functions and compared to closely matched healthy controls. Our finding that a problem of mental effort can cause subtle dysfunctioning may have been overlooked in the case studies, because no neuropsychological screening was carried out. In our view, tasks that require higher order cognitive processing beyond simple perceptual motor functioning or tasks that require subjects to work under time pressure caused problems for our study population. In addition, these problems tended to surface in tests in which subjects had to distribute their attention over more than one task.
The maximum score of both patients and controls on the Barthel index is not surprising as this scale has a ceiling effect and only captures physical domains of health status and the ability to function independently. Therefore, it turned out to be not adequate for assessing the impact of the consequences of a lacunar infarct. Another factor that can have a negative influence on quality of life is a change in emotional functioning after a lacunar infarct, either as a direct result of the specific brain lesion or as a secondary reaction to the effects of the lesion. The results of the short semistructured interview supported our assumption that most patients have emotional changes after a single lacunar infarct. From the five questions of the semistructured interview, the subjective experience of a change in emotional functioning showed the most marked difference between the patients and the controls. Surprisingly, the patients had no specific complaints about fatigue. Although our interview was brief, it indicated substantial emotional changes after a lacunar infarct.

In conclusion, the results of this study show that patients with a single supratentorial lacunar stroke can show subtle impairments on neuropsychological tasks. In addition, they seem to have subjective emotional problems. We conclude that these problems contribute to the experienced reduction of performance in everyday life.

1 Haan RJ de, Limburg M, Van der Meulen JHP, et al. Quality of life after stroke. Impact of stroke type and lesion location. Stroke 1995;26:402-8

2 Tatemichi TK, Desmond DW, Stern Y, et al. Cognitive impairment after stroke: frequency, patterns, and relationship to functional abilities. F Neurol Neurosurg Psychiatry 1994;57:202-7.

3 Fischer CM. Lacunar strokes and infarcts: a review. Neurology 1982;32:871-6.

4 Bogousslavsky J, Regli F, Uske A. Thalamic infarct: clinical syndromes, etiology and prognosis. Neurology 1988;38: 837-48.

5 Caplan LR, Schmahmann JD, Kase CS, et al. Caudate infarcts. Arch Neurol 1990;47:133-43.

6 Clavier I, Hommel M, Besson G, et al. Long-term prognosis of symptomatic lacunar infarcts. A hospital-based study. of symptomatic lacunar
Stroke 1994;25:2005-9.

Stroke 1994;25:2005-9.
7 Duncan PW, Samsa GP, Weinberger M, et al. Health status of individuals with mild stroke. Stroke 1997;7:740-5.

8 Shin AY, Porter PJ, Wallace MC, et al. Quality of life of stroke in younger individuals. Utility assessment in patients with arteriovenous malformations. Stroke 1997;28:2395-9.

9 Mahoney F, Barthel D. Functional evaluation: the Barthel Index. Maryland State Med F 1965;14:61-5.

10 Lezak MD. Neuropsychological assessment. 3th ed. Oxford: Oxford University Press, 1995

11 Muncer SJ, Jandreau S. Morphemes, syllables, words and reading. Percept Mot Skills 1984;1:14.

12 Robertson IH, Ward T, Ridgeway V, et al. The structure of normal human attention: the test of everyday attention. FINS 1996;2:525-34.

13 Brand N, Houx PJ. MINDS: towards a computerised test battery for health psychological and neuropsychological assessment. Behav Res Methods Instr Comp 1992;24:385-89.

14 Dimsdale JE. Reflections on the impact of antihypertensive medications on mood, sedation, and neuropsychologic medications on mood, sedation, and neurop
functioning. Arch Intern Med 1992;152:35-9.

15 Kahneman D. Attention and effort. Englewood Cliffs, New Jersey: Prentice-Hall, 1973

6 Damasio AR, Damasio H, Rizzo M, et al. Aphasia with nonhemorrhagic lesions in the basal ganglia and internal capsule. Arch Neurol 1982;39:15-20.

17 Basso A, Della Sala S, Farabola M. Aphasia arising from purely deep lesions. Cortex 1987;23:29-44.

18 Cappa SF, Cavallotti G, Guidotti M, et al. Subcortical aphasia: two clinical-CT scan correlation studies. Cortex 1983;19:227-41.

19 Naeser MA, Alexander MP, Helm-Estabrooks N, et al. Aphasia with predominantly subcortical lesion sites. Description of three capsular/putaminal aphasia synDescription of three capsular/putar

20 Tatemichi TK, Desmond DW, Prohovnik I, et al. Confusion and memory loss from capsular genu infarction: a thalamocortical disconnection syndrome? Neurology 1992;42: 1966-79. 
21 Schnider A, Gutbrod K, Hess W, et al. Memory without context: amnesia with confabulations after infarction of the right capsular genu. I Neurol Neurosurg Psychiatry 1996;61: 186-93.

22 Kooistra CA, Heilman KM. Memory loss from a subcortical white matter infarct. $\mathcal{F}$ Neurol Neurosurg Psychiatry 1988;51: $866-9$

23 Tanridag O, Kirshner HS. Aphasia and agraphia in lesions of the posterior internal capsule and putamen. Neurology 1985;35:1797-801.
24 Ferro JM, Kertesz A. Posterior internal capsule infarction associated with neglect. Arch Neurol 1984;41:422-4.

5 Pullicino P, Lichter D, Benedict R. Micrographia with cognitive dysfunction: minimal sequela of a putaminal infarct [case report]. Mov Disord 1994;3:371-3.

26 Yamanaka K, Fukuyama H, Kimura J. Abulia from unilateral capsular genu infarction: report two cases. $\mathcal{F}$ Neurol Sci 1996;143:181-4.

27 Verhage F, Van der Werff JJ. Een variantie-analyse op GIT-scores. Ned T Psychol 1964;19:497-509.

\section{Dorsolateral pontine segmental infarction presenting as isolated trigeminal sensory neuropathy}
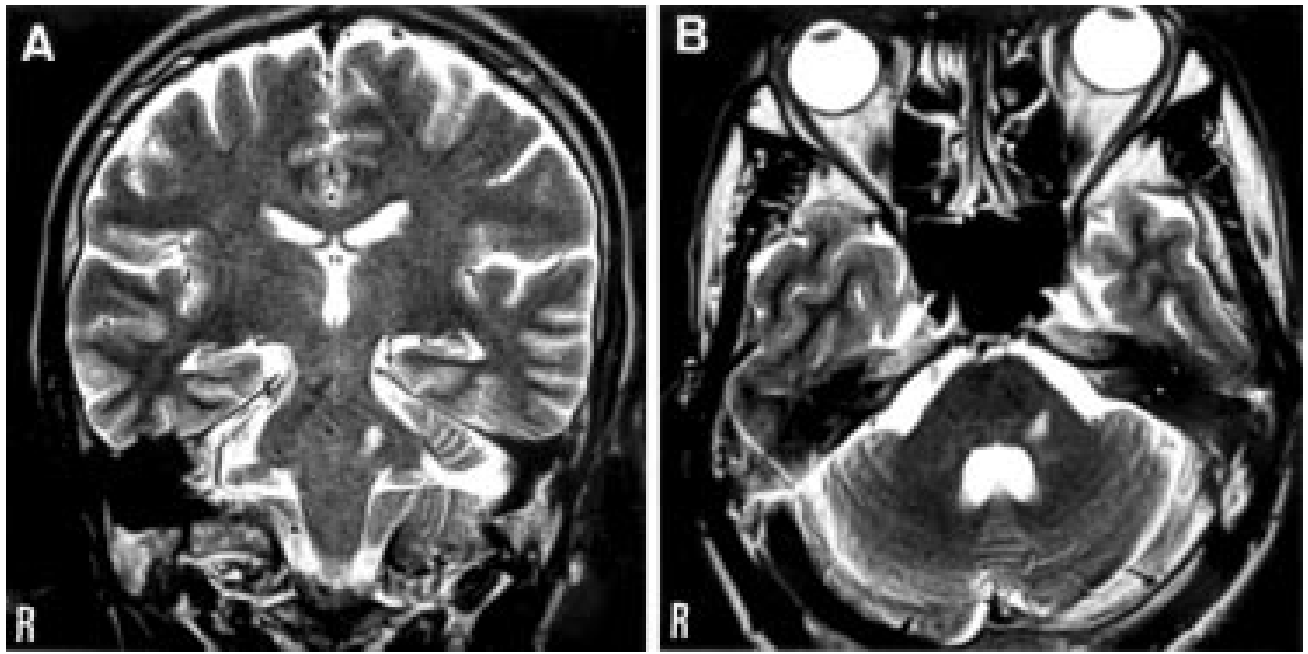

Small infarction in the left dorsolateral pontine tegmentum was identified by MRI in a 41 year old man presenting with isolated orofacial sensory deficits in the absence of other neurological findings.

Isolated trigeminal sensory neuropathy resulting from brain stem vascular lesions, either haemorrhagic ${ }^{1}$ or ischaemic, ${ }^{3}$ has been extremely rarely reported.

Our patient awoke to find numbness and paraesthesia over the left upper face, tongue, and buccal mucosa. Neurological examination disclosed non-dissociated sensory deficits on the same region. The corneal responsiveness was normal, and no hypotonus and weakness of the masseter muscle was detected. These findings implicated exclusive involvement of the pontine trigeminal sensory complex, including the principal sensory nucleus and pars oralis of the spinal trigeminal nucleus and tract.

The figure shows (A) the coronal and (B) the axial T2 weighted images, with a hypointense lesion in the left dorsolateral pontine tegmentum a few days after the onset.

\section{KAZUHIRO ISHII AKIRA TAMAOKA SHIN'ICHI SHOJI Department of Neurology, Institute of Clinical Medicine, University of Tsukuba, fapan}

Correspondence to: Dr Akira Tamaoka, Department of Neurology, Institute of Clinical Medicine, University of Tsukuba, 1-1-1 Tennoudai, Tsukuba, Ibaraki 305-0006, Japan.

1 Holtzman RNN, Zablozki V, Yang WC, et al. Lateral pontine segmental hemorrhage presenting as isolated trigeminal sensory neuropathy. Neurology 1987;37:704-6.

Kaneko K, Yuasa T, Miyatake T. Pontine infarction manifesting as isolated trigeminal sensory neuropathy. Neurol Med (Tokyo) 1990;32:526-8.

3 Nakamura K, Yamamoto T, Yamashita M. Small medullary infarction presenting as painful trigeminal sensory neuropathy. $\mathcal{F}$ Neurol Neurosurg Psychiatry 1996;61:138. 